\title{
HIPPARCOS AND PRIMARY DISTANCE SCALE INDICATORS
}

\author{
C. TURON \\ DASGAL, URA CNRS 335, Observatoire de Paris, 92195, Meudon Cedex, France.
}

\section{Introduction}

The Hipparcos data are providing a dramatic increase, qualitatively and quantitatively, of the basic available distance information. For example, the numbers of stars for which a relative accuracy better than $10 \%$ is available from Hipparcos data and from ground-based data are respectively 22396 and about 1000 . Moreover, the range of spectral types and luminosity classes for which precise parallaxes are available is considerably enlarged, including many stars in open clusters and a small number of Cepheids and RR Lyrae. The bottom of the main sequence is populated down to absolute $H p$ magnitude 14, including a number of subdwarf stars essential to derive globular clusters distances and ages (Pont et al., 1997a).

Finally, the Hipparcos data show how difficult are the calibration of photometric distances and the transformation of relative trigonometric parallaxes to absolute parallaxes. This is illustrated by the comparison of distances given in the last edition of the Catalogue of Nearby Stars (CNS3, Gliese \& Jahreiß 1991), which is the best available compilation of stars said to be closer than 25 pc from ground-based data. About a third of them are found by Hipparcos to be (much) further than this limit (Perryman et al., 1995). A second example is given by the study of (Binney et al., 1997).

\section{Open clusters}

Open clusters are widely used for distance determination by main sequence fitting. Hipparcos offers for the first time the possibility of thoroughly studying the three-dimensional structure and the kinematics of the closest cluster, the Hyades, giving a mean distance modulus of $3.33 \pm 0.01 \mathrm{mag}$ for the stars observed by Hipparcos within 10 pc of the cluster center (Perryman et al., 1997a), and to precisely place the main sequences of about 15 open clusters of various ages and chemical compositions in the HR diagram (Mermilliod et al., 1997), (Robichon etal., 1997), (van Leeuwen \& Hansen Ruiz, 1997). The surprise is that the positions of the different sequences cannot be explained by differences in metallicities, and that a scatter of Helium abondance of about 0.05 would be necessary to explain these discrepancies. A further use of the main-sequence fitting method requires the understanding of this problem.

\section{Pulsating variables}

Pulsating variables are some of the more powerful objects for distance determinations, after proper calibration of period-(colour-metallicity)-luminosity relations. The main difficulty is to obtain a representative sampling of the stars considered, covering the whole period, colour, and metallicity ranges for each type of objects. A few trigonometric parallaxes, good proper motions and improved cluster membership for Cepheids are obtained from Hipparcos data. Various authors have presented period-luminosity relations: (Feast \& Catchpole, 1997), (Feast \& Whitelock, 1997), (Paturel et al., 1997), (Szabados, 1997), (Madore \& Freedman, 1997) and (Luri et al., 1997) for Cepheids; (Fernley et al., 1997), (Gratton et al., 1997), (Reid, 1997), (Tsujimoto etal., 1997), (Feast \& Whitelock, 1997), (Chaboyer et al., 1997), and (Luri et al., 1997) for RR Lyrae. There is still a scatter in the results, easily explained by the small number of stars used in each study. 
More photometric observations are strongly needed to better evaluate the intrinsic scatter in period-luminosity relations, the interstellar extinction, and the metallicities. However, only a project such as Gaia (Lindegren \& Perryman, 1997), (Perryman et al., 1997b) would bring extremely accurate determinations of absolute magnitudes for extensive samples of pulsating variable stars and allow a comprehensive calibration of period-luminosity relations for all galactic (and even the brightest Magellanic) variables of the various stellar populations (both disk and halo).

\section{Results and conclusion}

The distance of the Large Magellanic Cloud and the age of globular clusters have been derived by various authors, from the Hipparcos data of several types of objects, and using many other groundbased observations. The results are given below. Most results are converging to a LMC distance modulus of 18.5-18.7, but the only paper with no discrepancy between the distance obtained from RR Lyrae and from Cepheids (Luri et al., 1997) gives about 18.3. For the age of globular clusters, there is some spread in the results, but most are converging to 12-14 Gyr. These results go towards solving the previous discrepancy between the age of the Universe, and the age of the oldest objects.

To go further, many more very accurate distances are needed, but also many more accurate photometric and spectroscopic observations, and more detailed theoretical models.

\begin{tabular}{|c|c|c|c|c|}
\hline \multicolumn{2}{|c|}{ LMC distance modulus } & \multicolumn{3}{|c|}{ Globular cluster age } \\
\hline Objects/Authors & $(M-m)_{0}$ & Objects/Authors & Age & (Gyr) \\
\hline $\begin{array}{l}\text { from Cepheids } \\
\text { (Feast \& Catchpole, 1997) } \\
\text { (Paturel et al., 1997) } \\
\text { (Madore \& Freedman, 1997) } \\
\text { (Luri et al., 1997) (1) } \\
\text { (Luri et al., 1997) (2) }\end{array}$ & $\begin{array}{l}18.70 \pm 0.10 \\
18.7 \\
18.57 \pm 0.11 \\
18.29 \pm 0.17 \\
18.21 \pm 0.20\end{array}$ & $\begin{array}{l}\text { from subdwarfs } \\
\text { (Reid, 1997) } \\
\text { (Gratton et al., 1997) } \\
\text { (Pont et al., 1997b) } \\
\text { (Cayrel et al., 1997) }\end{array}$ & $\begin{array}{l}12-13 \\
11.8 \\
14 \\
14-15\end{array}$ & $\begin{array}{l}+2.1 \\
-2.5 \\
\pm 1\end{array}$ \\
\hline $\begin{array}{l}\text { from RR Lyrae } \\
\text { (Fernley et. al., 1997) } \\
\text { (Luri et al., 1997) }\end{array}$ & $\begin{array}{l}18.31 \pm 0.10 \\
18.37 \pm 0.23\end{array}$ & $\begin{array}{l}\text { various methods + RR Ly } \\
\text { (Chaboyer et al., 1997) }\end{array}$ & $\begin{array}{l}\text { yrae } \\
11.78\end{array}$ & \pm 1.4 \\
\hline $\begin{array}{l}\text { from RR Lyrae, via subd } \\
\text { (Reid, 1997) } \\
\text { (Gratton et al., 1997) }\end{array}$ & $\begin{array}{l}\operatorname{arfs} \\
18.65 \\
18.60 \pm 0.07\end{array}$ & $\begin{array}{l}\text { via RR Lyrae } \\
\text { (Fernley et al., 1997) } \\
\quad \text { using (Chaboyer et al., } 1997\end{array}$ & $\begin{array}{l}\text { 15.8 } \\
\text { 7) } \mathrm{Mv} \text { for }\end{array}$ & RR Lyrae \\
\hline $\begin{array}{l}\text { from Mirae } \\
\text { (van Leeuwen et al., 1997) } \\
\text { (Whitelock etal., 1997) }\end{array}$ & $\begin{array}{l}18.54 \pm 0.18 \\
18.60 \pm 0.18\end{array}$ & $\begin{array}{l}\text { via Cepheids } \\
\text { (Feast \& Whitelock, 1997) }\end{array}$ & 11 & \\
\hline
\end{tabular}

\section{References}

Binney, J., Dehnen, W., Houk, N., Murray, C.A., Penston, M.J., (1997), Hipparcos Venice '97, ESA SP-402, p. 473

Cayrel, R., Lebreton, Y., Perrin, M.N., Turon, C. (1997), Hipparcos Venice '97, ESA SP-402, p. 219

Chaboyer, B., Demarque, P., Kernan, P.J., Krauss, L.M. (1997), ApJ, astro-p/9706128

Feast, M.W., Catchpole, R.M., (1997), MNRAS, 286, L1

Feast, M.W., Whitelock, P.A., (1997), Hipparcos Venice 'g7, ESA SP-402, p. 625

Fernley, J., Barnes, T.G., Skillen, I., et al., (1997), Hipparcos Venice '97, ESA SP-402, p. 635

Gliese, W., Jahreiß, H. (1991), Astron. Rechen-Institut, Heidelberg

Gratton, R.G., Fusi Pecci, F., Carretta, E., Clementini, G., et al. (1997), Hipparcos Venice '97, ESA SP-402, p. 651

van Leeuwen, F, Hansen Ruiz, C.S. (1997), Hipparcos Venice '97, ESA SP-402, p. 689

van Leeuwen, F., Feast, M.W., Whitelock, P.A., Yudin, B, (1997), MNRAS, 287, 955

Lindegren, L., Perryman, M.A.C. (1997), Hipparcos Venice 'g7, ESA SP-402, p. 799

Luri, X., Gómez, A.E., Torra, J., Figueras, F., Mennessier, M.O. (1997), Astron. Astrophys. Letters, submitted

Madore, B.F., Freedman, W.L. (1997), ApJ Letters, in press

Mermilliod, J.C., Turon, C., Robichon, N. et al. (1997), Hipparcos Venice '97, ESA SP-402, p. 643

Paturel, G., Lanoix, P., Garnier, R., et al., (1997), Hipparcos Venice '97, ESA SP-402, p. 629

Perryman, M.A.C., Brown, A.G.A., Lebreton, Y. et al. (1997a), Astron. Astrophys., in press

Perryman, M.A.C., Lindegren, L., Turon, C. (1997b), Hippareos Venice '97, ESA SP-402, P. 743

Perryman, M.A.C., Lindegren, L., Kovalevsky, J., Turon, C., Høg, E., et al. (1995), Astron. Astrophys., 304, 69

Pont, F., Mayor, M., Turon, C. (1997a), JAU Symp. 189, T.R. Bedding et al. (eds.), p. 433

Pont, F., Charbonnel, C., Lebreton, Y. et al. (1997b), Hipparcos Venice '97, ESA SP-402, p. 699

Reid, N. (1997), Astron. J, 114, 161

Robichon, N., Arenou, F., Turon, C., et al. (1997), Highlights in Astronomy, 11, in press

Szabados, L. (1997), Hipparcos Venice '97, ESA SP-402, p. 661

Tsujimoto, T., Miyamoto, M., Yoshii, Y., (1997), Hipparcos Venice '97, ESA SP-402, p. 639

Whitelock, P.A., van Leeuwen, F., Feast, M.W. (1997), Hipparcos Venice '97, ESA SP-402, p. 213 